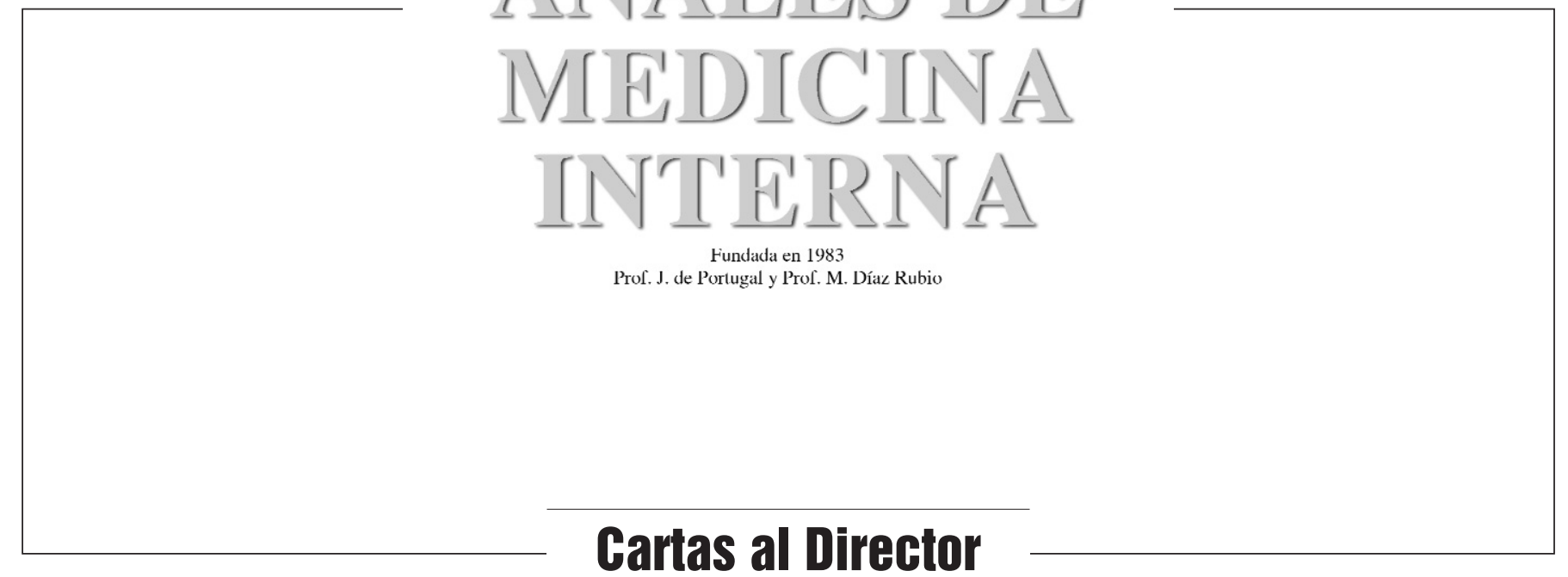

\section{Pseudomixoma peritoneal de origen desconocido}

\section{Sr. Director:}

El pseudomixoma peritoneal se caracteriza por ascitis mucinosa con implantes peritoneales epiteliales y mucinosos secundarios a ruptura ó metástasis de un tumor primario. Es una enfermedad poco frecuente, con una incidencia aproximada de 2 casos por cada 10.000 laparotomías $(1,2)$. Se asocia con frecuencia a tumores apendiculares y ováricos. En raras ocasiones, 5\% de los casos, el origen es desconocido (1-3). El diagnóstico se suele realizar por laparoscopia. Actualmente existen controversias sobre el protocolo de tratamiento a seguir, aunque la mayoría de los autores consideran como primera elección la cirugía (1-7). Presentamos un caso de pseudomixoma peritoneal de origen desconocido.

Se trata de un varón de 67 años sin antecedentes personales de interés. Consulta por presentar desde hace 3-4 meses dolor abdominal generalizado y sensación de distensión a nivel de ambos hipocondrios, flancos y epigastrio. Acompañado de náuseas, discreta pérdida de peso no cuantificada y anorexia. No había presentado vómitos, cambios en el hábito intestinal, disnea, fiebre, ni otros síntomas. El paciente se encontraba afebril, sin adenopatías periféricas objetivables. En la exploración abdominal destacaba semiología de ascitis, sin masas ni organomegalias palpables. El resto de la exploración no presentaba hallazgos significativos. El hemograma, la coagulación y la bioquímica eran normales, con velocidad de sedimentación de $90 \mathrm{~mm}$. Se realizó paracentesis de líquido ascítico en el que se obtuvo hties $350 / \mathrm{mm}^{3}$, leucocitos $15 / \mathrm{mm}^{3}$, glucosa $38 \mathrm{mg} / \mathrm{dl}$, proteínas totales $3,9 \mathrm{~g} / \mathrm{dl}$, lacticodeshidrogenasa $703 \mathrm{UI} / \mathrm{L}$, adenosindeaminasa $27,4 \mathrm{UI} / \mathrm{L}$, los cultivos y citologías realizados del mismo se informaron como negativos para patología infecciosa y células tumorales malignas. Se realizaron ecografía y tomografía axial computerizada con PAAF de líquido ascítico informadas como ascitis masiva multitabicada, mucinosa e implantes peritoneales metastásicos, sin apreciar lesión visceral que justificara los hallazgos. La gastroscopia y colonoscopia realizadas no presentaron alteraciones significativas. Se decidió realizar laparoscopia diagnóstica en la que se objetivaron múltiples adherencias y moco espeso de distintas densidades en cavidad abdominal. Se tomó biopsia de los implantes, cuyo informe anatomopatológico fue tumor mucinoso de bajo grado de malignidad, compatible con pseudomixoma peritoneal. Tras ser valorado por el servicio de oncología y de acuerdo con el paciente se decidió realizar sólo tratamiento quirúrgico paliativo. En revisiones posteriores, tras 20 meses del inicio de la sintomatología, el paciente continúa en situación estable.

Los tumores primarios que lo ocasionan con mayor frecuencia son el carcinoma de apéndice (52\%), ovario (34\%) y colon (4\%). Sólo en el 5\% de los casos no se encuentra el origen, como sucedió en nuestro caso. Suele aparecer en la sexta década de la vida, siendo más habitual en el sexo femenino (64-75\%) (1-3). La clínica es inespecífica, provocando síntomas digestivos, ginecológicos ó urológicos. Las manifestaciones más frecuentes son dolor, distensión abdominal, anorexia y náuseas. Suelen consultar por la presencia masa o distensión abdominal. La complicación más frecuente es la obstrucción intestinal que cursa con gran morbimortalidad.

La ecografía y la tomografia axial computerizada ayudan al diagnóstico preoperatorio. Se puede apreciar desplazamiento de asas intestinales, compresión extrínseca de vísceras, irregularidades en el borde hepático e imágenes quísticas con septos ó tabiques (6). No obstante, la mayoría de los casos se diagnostican mediante laparoscopia confirmado por la anatomía patológica (17). La presentación anatomopatológica más frecuente es el adenocarcinoma (64\%) seguida del cistoadenocarcinoma (32\%), en el $4 \%$ de los casos es inclasificable (1).

No existe consenso en cuanto al tratamiento, la mayoría de los autores consideran de primera elección la cirugía, utilizada de forma paliativa para reducir el tamaño tumoral, acompañada de quimioterapia intraperitoneal. Otras opciones de tratamiento adyuvante son la quimioterapia sistémica, la radioterapia intraperitoneal, el tratamiento fotodinámico intraoperatorio y las instilaciones de dextrosa al 5\% (1-4,7). El curso de la enfermedad independientemente del tratamiento empleado es maligno, presentando un $76 \%$ de recidivas a los 12 años, el crecimiento recurrente ocasiona problemas por efecto masa, obstrucción intestinal y fístulas que pueden conducir al paciente a la muerte (1).

\section{D. Mañas García, C. Lázaro Bermejo, J. Gijón Rodríguez, R. Pardo García ${ }^{1}$}

Servicio de Medicina Interna $y{ }^{\prime}$ Cirugía General. Complejo Hospitalario. Ciudad Real 
1. Sánchez LA, Franco-Vicario R, Ferrero OL, Pérez MJ, Pueyo V, Solano D, et al. Pseudomixoma peritoneal. Rev Clin Espl 1995; 195: 57-62.

2. Sherer DM, Abulafia O, Eliakim R. Pseudomixoma peritonei: a review of current literature. Gynecol Obstet Invest 2001; 51: 73-80.

3. Marco C, Veloso E, Muñoz E, Bretcha P, Rodríguez-Santiago J, Casalots $\mathrm{J}$, et al. Pseudomixoma peritoneal de origen apendicular. Cir Esp 2000; 68: 464-466.

4. Jivan S, Bahal V. Pseudomixoma peritonei. Postgrad Med J 2002; 78 : 170-172.

5. Baradi H, Ponsky J. Pseudomixoma peritonei. Surgery 2002;131:230231.

6. Guillarte J, Cantero J, Mantas JA. Hallazgos ecográficos y tomográficos en el pseudomixoma peritoneal. Rev Esp Enferm Dig 1997;89:798-799.

7. Sugarbaker PH. Cytoreductive surgery and peri-operative intraperitoneal chemotherapy as a curative approach to pseudomyxoma peritonei syndrome. Eur J Surg Oncol 2001; 27: 239-243.

\section{Hematoma abdominal secundario al empleo de nadroparina}

\section{Sr. Director:}

El empleo de heparinas de bajo peso molecular (HBPM) a dosis terapeútica se ha convertido en tratamiento habitual en la cardiopatía isquémica aguda, tipo angor inestable e infarto de miocardio (IAM) sin y con elevación del segmento ST, como alternativa a la heparina sódica (1-3), con suficiente evidencia de una eficacia igual $(4,5)$ e incluso superior a la heparina no fraccionada (6). Efectivamente los últimos estudios publicados establecen claramente las ventajas de las HBPM, fundamentalmente por su mayor inhibición de la formación de trombina, perfil farmacocinético predecible, alta biodisponibilidad y larga vida media, lo cual resulta en niveles efectivos de actividad anticoagulante después de la administración subcutánea, sin necesidad de controles hematológicos. No obstante sus complicaciones hemorrágicas, pese a la baja incidencia descrita $(4,6-9)$, pueden acontecer y ser severas especialmente en tratamientos prolongados (5).

Presentamos el caso de una paciente tratada con dosis terapéuticas de HBPM, que se complica con gran hematoma de pared abdominal. Mujer de 72 años, con antecedentes de cardiopatía isquémica tipo angor, HTA, nefrectomía derecha y asma, que ingresa por angor inestable, iniciándose tratamiento con nitroglicerina IV, calcioantagonistas, ticlopidina y nadroparina a dosis terapeútica ajustada al peso; pese al tratamiento persisten los episodios anginosos, con cambios en ECG, por lo que se efectúa cateterismo que muestra enfermedad coronaria de tres vasos con fracción de eyección conservada, quedando pendiente de sesión médico-quirúrgica. Posteriormente evoluciona a IAM no Q, tras lo cual queda asintomática para angor. Veinticuatro horas después del IAM, tras trece días de tratamiento anticoagulante, presenta bruscamente dolor en fosa ilíaca y vacío derechos, una TAC abdominal muestra engrosamiento de músculo recto anterior, debido a la presencia de una gran colección con nivel líquido-líquido en su interior que se comunica con el espacio prevesical, que se interpretó como sangrado a dicho nivel y menos probablemente como relacionado con patología ginecológica subyacente; en ese momento la enferma mantiene cifras de hemoglobina y está hemodinamicamente estable, pero en horas sucesivas entra en shock con severa anemización ( $\mathrm{Hb} 5,3 \mathrm{~g} / \mathrm{dl}$ ) sin coagulopatía, por lo que se interviene de urgencia con sospecha de quiste ovárico torsionado, encontrándose gran hematoma de pared de aproximadamente un litro, sin claro punto sangrante y con cavidad abdominal normal. La paciente presenta postoperatorio sin complicaciones siendo finalmente alta hospitalaria.
Consideramos que las HBPM tienen actualmente clara indicación en el tratamiento del síndrome coronario agudo, con sobrado aval en cuanto a su eficacia y seguridad, incluso con ventajas sobre la heparina sódica, pero debemos recordar que, aunque infrecuentes, existe con ellas riesgo de complicaciones hemorrágicas potencialmente graves, especialmente en tratamientos superiores a una semana.

\section{R. M. García Fanjul, M. T. Antuña Braña, J. A. Lapuerta Irigoyen, M. J. Gutiérrez Fernández}

Servicio de Medicina Intensiva. Hospital de Cabueñes de Gijón. Asturias

1. Braunwald E, Antman EM, Beasley JW, Califf RM, et al. ACC/AHA guidelines for the manegement of patients with unstable angina and non-ST-segment elevation myocardial infarction: executive summary and recommendations: a report of the American College of Cardiology/American Heart Association Task Force on Practice Guidelines(Committee on Manegement of Patients With Unstable Angina). Circulation 2000; 102: 1193-1209.

2. López L, Fernández-Ortiz A, Bueno H, Coma I, et al. Guías de práctica clínica de la Sociedad Española de Cardiología en la angina inestable/infarto sin elevación ST. Rev Esp Cardiol 2000; 53: 838-850.

3. Arós F, Heras M. Tendencias futuras del tratamiento del síndrome coronario agudo. Papel de las heparinas de bajo peso molecular. Rev Esp Cardiol Supl 2001; 1: 76B-83B.

4. Swahn E and Wallentin L for the FRISC Study Group. Low-molecularweight heparin (Fragmin) during instability in coronary artery disease (FRISC). Am J Cardiol 1997; 80 (5A): 25E-29E.

5. The FRAXIS study group. Comparison of two treatment durations ( 6 days and 14 days) of a low molecular weight heparin with a 6-day treatment of unfractioned heparin in the initial management of unstable angina or nonQ wave myocardial infarction: FRAXIS (FRAXiparine in Ischaemic Syndrome). Eur Heart J 1999; 20: 1553-1562.

6. Cohen M, Demers C, Gurfinkel EP, G Turpie AG, Fromell GF, Goodman $\mathrm{S}$, et al for the ESSENCE study group. Low-molecular-weight heparins in non-ST-segment elevation ischemia: the ESSENCE trial. Am J Cardiol 1998; 82: 19L-24L.

7. Klein W, Buchwald A, Hillis S, Monrad S, Sanz G, G Turpie AG, et al. Fragmin in unstable angina pectoris or in non-Q-wave acute myocardial infarction (the FRIC study). Am J Cardiol 1997; 80 (5A): 30E-34E.

8. Gómez de la Torre R, Milla Crespo A, Cadenas F, Fernández Bustamante J, Vázquez Castañón M. Hematoma retroperitoneal espontáneo inducido por enoxaparina a dosis terapeúticas. An Med Interna (Madrid) 2003; 20 (7): 386-87.

9. Yusty Campo M, Lado Lado FL, Pérez Herbón M, Rodríguez Constenla I, Rodríguez López I. Hematoma de pared abdominal como complicación del uso de heparinas de bajo peso molecular. An Med Interna (Madrid) 2003; 20 (5): 273.

\section{Mujer de 26 años con náuseas y distensión abdominal}

\section{Sr. Director:}

Paciente mujer de 26 años diagnosticada de anorexia nerviosa desde hace aproximadamente 6 años que acude al servicio de urgencias por cuadro de 24 horas de evolución de sensación de plenitud gástrica, náuseas sin vómitos, con última deposición de características normales hace 6 horas. Refiere importante ingesta de fruta y soja varias horas previas al cuadro clínico.

En la exploración física destaca la presencia de una mujer caquéctica con un abdomen muy distendido, timpánico a la percusión y difusamente doloroso a la palpación profunda sin peritonismo, pero con ruidos hidroaéreos aumentados. Se realizó radiografía de abdomen simple (Fig. 1) encontrándose un patrón en miga 

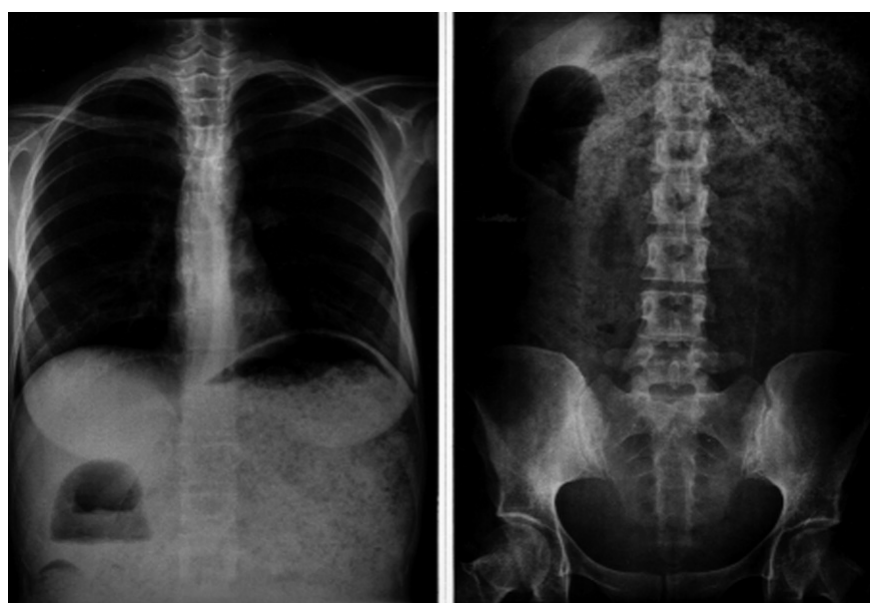

Fig. 1. Radiografía simple: patrón en miga de pan distribuido por todo el abdomen con niveles hidroaéreos que parecen corresponder al antro gástrico y marco duodenal.

de pan distribuido por todo el abdomen, delimitándose perfectamente la curvadura menor del estómago y dos niveles hidroareos que parecen corresponder al antro gástrico y al marco duodenal. Ante la sospecha de bezoar se solicitó endoscopia (Fig. 2) que demostró un estómago muy dilatado, con abundantes restos alimentarios (fibra vegetal) que ocupa la mayor parte de la cavidad gástrica, formando una masa suelta, no extraíble endoscópicamente. Se observa en curvadura menor y cara posterior de cuerpo múltiples erosiones, y pequeñas ulceraciones superficiales de la mucosa. Debido a la dilatación gástrica, se alcanza duodeno, pero se visualiza píloro que aparece abierto y permeable.

Diagnóstico: fitobezoar gástrico y múltiples erosiones y úlceras gástricas por decúbito.

El término "bezoar" procede del vocablo persa "padzahr", cuya traducción literal corresponde a "contraveneno", sin embargo, en terminología médica se utiliza para designar a cuerpos extraños formados por la concreción de vegetales, pelos o alimentos digeridos en el tubo digestivo. La cirugía previa (sobre todo vagotomía troncular y piloroplastia), la gastroparesia diabética y alteraciones de la masticación son factores predisponentes para su génesis, aunque como en el caso que nos ocupa los trastornos mentales de la alimentación también pueden contribuir a su formación, especialmente en el caso de los tricobezoares (concreciones de pelos).

La forma de presentación es muy variable y puede simular cualquier tipo de patología abdominal, aunque el dolor abdominal es el síntoma principal, al que se pueden asociar nauseas, vómitos y distensión abdominal.

En cuanto al diagnóstico, la radiografía simple de abdomen muestra la presencia de un estómago muy dilatado con restos alimentarios en su interior, que puede verse como un "patrón moteado", y si el fitobezoar está impactado en el yeyuno o en el íleon signos de obstrucción de intestino delgado. El tránsito baritado y la ecografía son otros métodos muy útiles para su diagnóstico.

El tratamiento es esencialmente quirúrgico aunque la gastroscopia puede conseguir ocasionalmente su extracción (no así en el caso que nos ocupa en el que fue necesario la gastrotomía anterior para proceder a su extracción).

J. Hinojosa Mena-Bernal, C. Hinojosa Mena-Bernal, S. Martín Santos, C. Cuervo Molinero, F. Arranz, D. Llanos Pérez ${ }^{1}$

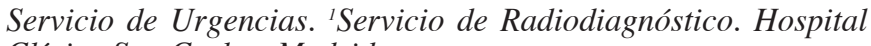
Clínico San Carlos. Madrid
1. Martín Parra JI, Sandoval González F, de Diego García EM, Naranjo Gómez A. An Esp Pediatr 1998; 48: 76-8.

2. Pedrosa CS, Casanova R. Diagnóstico por imagen: Tratado de radiología clínica. De. McGraw-Hill-Iberoamericana 2000; 367: 475-741.

\section{Espondilodiscitis y absceso de psoas por Escherichia coli}

\section{Sr. Director:}

Tras la lectura del trabajo publicado en su revista "Osteomielitis vertebral por $E$. coli" (1), queremos aportar otro caso de espondilodiscitis infecciosa por Escherichia coli.

Mujer de 72 años con antecedentes personales de diabetes mellitus, insuficiencia mitral y fibrilación auricular. Consultó por síndrome miccional y dolor en fosa lumbar derecha de características mecánicas irradiado a rodilla con impotencia funcional sin antecedente traumático. En la exploración física destacaban obesidad, signos de deshidratación y temperatura de $39,5^{\circ} \mathrm{C}$. La auscultación cardiaca era arrítmica y presentaba un soplo sistólico mitral. La auscultación pulmonar era normal. El abdomen estaba distendido y poco depresible, doloroso a la palpación en fosa iliaca izquierda, sin signos de irritación peritoneal. La puñopercusión renal bilateral era negativa. El signo de Lassegue era positivo a $30^{\circ}$. La fuerza, la sensibilidad y los reflejos osteotendinosos estaban conservados en las cuatro extremidades.

Pruebas complementarias: destacaban en el hemograma: leucocitos $22.000 / \mathrm{mL}$ con neutrofilia. Bioquímica: creatinina 1,9 $\mathrm{mg} / \mathrm{dL}$, urea $79 \mathrm{mg} / \mathrm{dL}$, Na $127 \mathrm{mEq} / \mathrm{L}$ y K 3,6 mEq/L. Sedimento urinario: piuria de moderada intensidad. Radiografía de tórax: cardiomegalia, no infiltrados agudos. Radiografía de abdomen: patrón hidroaéreo normal, escoliosis lumbar y osteopenia radiológica. Tomografía axial computarizada (TAC) abdominal: absceso de psoas derecho. Se sacaron hemocultivos: bacilo gram negativo (más tarde identificado como Escherichia coli). Por todo ello, se interpretó el cuadro como bacteriemia por E. coli de origen urinario que secundariamente colonizó el psoas y se instauró tratamiento con amoxicilina-clavulánico.

La clínica abdominal mejoró pero persistía el dolor lumbar irradiado a miembro inferior derecho. La Velocidad de Sedimentación Globular (VSG) fue aumentando progresivamente hasta llegar a $91 \mathrm{~mm} / \mathrm{h}$ aunque la leucocitosis iba remitiendo. Tras 4 días de tratamiento antibiótico con amoxicilina-clavulánico se aisló E. coli resistente a dicho antibiótico en el urocultivo por lo que se cambió a cefotaxima. Ante la persistencia de dolor lumbar incapacitante, se realizó resonancia magnética nuclear (RMN) de columna en la que se objetivó una espondilodiscitis de L4-L5, una miositis de la musculatura extensora y dos abscesos en los músculos psoas. Se mantuvo el tratamiento con cefotaxima durante 8 semanas, durante las cuales mejoró la sintomatología, disminuyó la VSG y hubo mejoría radiológica.

Las espondilodiscititis bacterianas son procesos poco frecuentes. Su incidencia real es desconocida (2) Suelen presentarse como dolor continuo, rigidez y, en ocasiones, fiebre, así como VSG elevada y leucocitosis. Por orden de frecuencia se localizan en columna lumbar, dorsal y cervical (3). Puede llegar a existir incluso afectación neurológica.

La asociación entre absceso de psoas y espondilodiscitis lumbar por bacilo gram negativo es una entidad muy rara (4). Entre los factores de riesgo para la aparición de absceso de psoas primario se encuentran la diabetes, la inmunosupresión y la insuficiencia renal. Existen una gran variedad de causas de absceso secundario entre las que se encuentran la infección del tracto uri- 
nario, diverticulitis, apendicitis, espondilodiscitis lumbar, endocarditis, hemodiálisis, instrumentación e intervenciones quirúrgicas (5). En nuestro caso se daban varios factores de riesgo de los descritos, se trataba de una paciente diabética y con infección urinaria en la que coexistían absceso de psoas y espondilodiscitis.

Escherichia coli causa el 7\% aproximadamente de las espondilodiscitis infecciosas, por detrás de estreptococos, estafilococos y el bacilo tuberculoso (3).

Para el diagnóstico es necesario un alto grado de sospecha, ya que la clínica es muy inespecífica. Las técnicas de imagen, en especial la RMN confirman el diagnóstico. Los hemocultivos son positivos en un alto porcentaje de los casos, y no suelen ser necesarias medidas más agresivas para la identificación del germen. El tratamiento antibiótico intravenoso debe instaurarse lo más pronto posible en las espondilodiscitis infecciosas y debe mantenerse al menos durante 6 semanas (6).

\section{R. Torres-Gárate, E. Álvarez-Rodríguez, A. Gutiérrez Larrain- zar, M. A. Lozano, F. Fernández Mendieta, J. Cabello}

\author{
Servicio de Medicina Interna. Hospital Clínico San Carlos. \\ Madrid
}

1. Marcos F, Aragón A, Árbol F, Albo I, Viana A. Osteomielitis vertebral por E. Coli. An Med Interna 2004; 21: 43-44.

2. Muffoletto AJ, Detonen LM, Mader JT, Crow WN, Hadjipavlou AG. Hematogenous Pyogenic Facet Joint Infection. Spine 2001; 26: 157076.

3. Rivero MG, Salvatore AJ, de Wouters L. Spontaneous infectious spondylodiscitis in adults. Analysis of 30 cases. Medicina (B Aires) 1999; 59 (2): 143-50.

4. Ampudia-Blasco FJ, Fernández J, Ferrer MD, Pallardo Y, Tenes S, Carmena R. Psoas abscess secondary to lumbar spondylodiscitis caused by gram negative bacilli. An Med Interna (Madrid) 1998; 15: 436-38.

5. Babafemi T. Psoas Abscess: A primer for the internist. South Med J 2001; 94 (1): 2-5.

6. Ariza J, Gomis M, Barberán J, Sánchez C, Barros C. Infecciones osteoarticulares y de partes blandas.Protocolos clínicos SEIMC [revista electrónica] [Consultado 05-04-2004]: Disponible en: http://www.seimc.org /protocolos/clinicos/index.htm.

\section{Metástasis pulmonares de crecimiento lento con probable origen de carcinoma tiroideo diferenciado}

\section{Sr. Director:}

El carcinoma tiroideo supone el $1 \%$ de todos los cánceres, apareciendo en el $0,5 \%$ de los canceres en el varón y del 1-5\% en la mujer (1). En el estudio1 realizado en al zona sur de Madrid se observó que en la mujer tenía una incidencia del 4,6/100.000 al año.

El 94\% de los cánceres de tiroides son diferenciados. Cuando se habla de cáncer diferenciado, se refiere a los carcinoma papilar o folicular, que generalmente son curables cuando se diagnostican en una etapa temprana. Las tasas de supervivencia a los 10 años en los cánceres papilar y folicular son del $93 \%$ y del $85 \%$ respectivamente (2).

Mujer de 82 años con prolapso uterino, a la que se le detectan en control rutinario de radiografía de tórax múltiples nódulos pulmonares, no presentado sintomatología respiratoria alguna. Como antecedentes destacaban bocio de 37 años de evolución, por el que no había realizado ningún seguimiento; e infecciones respiratorias frecuentes. En la exploración física solo destacaba un bocio heterogéneo a la palpación con una zona más dura y no adherida en el lóbulo izquierdo, no presentaba adenopatías ni visceromegalias, siendo la auscultación cardiaca anodina. En cuanto a las pruebas complementarias el hemograma era normal, la VSG era de 32, y la función era renal y las transaminasas también eran normales. Hormonas tiroideas normales. Tiroglobulina 2.745 $\mathrm{ng} / \mathrm{mL}$ (límite de referencia hasta $25 \mathrm{ng} / \mathrm{mL}$ ). La radiografía de tórax (Fig. 1) mostraba una masa en mediastino antero-superior que desplazaba tráquea hacia la izquierda y la obliteraba parcialmente, nódulos pulmonares múltiples bilaterales de contornos nítidos. En la TAC torácica aparecía un bocio multinodular que comprimía tráquea, esófago y troncos supraaórticos; y nódulos pulmonares múltiples de bordes bien definidos. La gammagrafía mostraba una imagen compatible con un bocio multinodular, con un nódulo frío de considerable tamaño que ocupaba la totalidad del lóbulo derecho que presentaba bordes irregulares. Se practicaron PAAF de nódulo tiroideo y punción por TAC de los nódulos pulmonares que no fueron diagnósticos. Con los datos obtenidos se realizó el diagnóstico de probable carcinoma diferenciado de tiroides con metástasis pulmonares. A petición familiar no se administró ningún tratamiento y la paciente no siguió más controles.

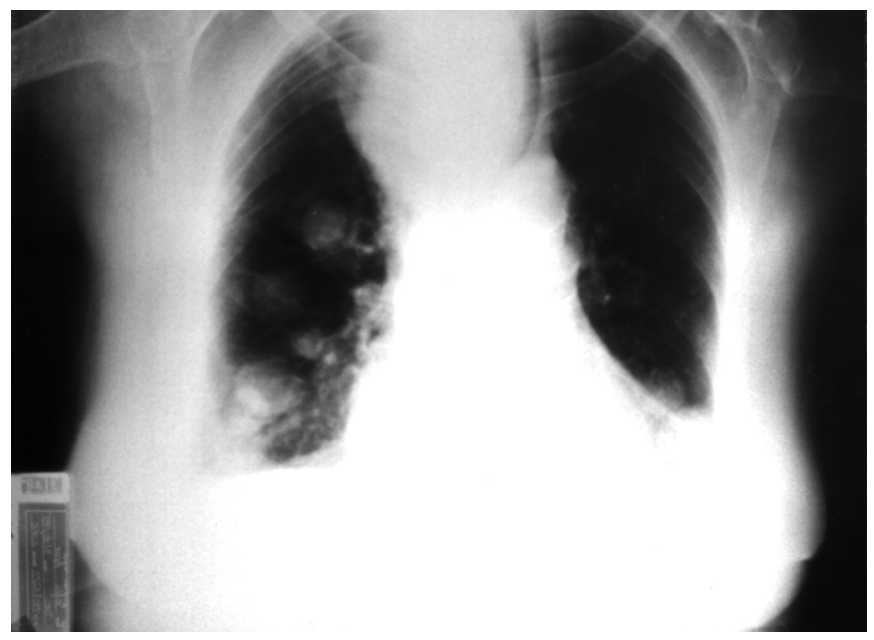

Fig. 1. Radiografía de tórax en la que aparece una masa en mediastino antero-superior que desplaza tráquea hacia la izquierda y la oblitera parcialmente, nódulos pulmonares múltiples bilaterales de contornos nítidos.

Cuando la paciente contaba con 93 años de edad, la consultó por aumento de su disnea habitual. Se realizó radiografía de tórax y TAC torácica, apareciendo un aumento en el tamaño y número de los nódulos pulmonares. La paciente falleció debido al compromiso aéreo producido por las metástasis pulmonares.

Debido a que el tejido tiroideo es la única fuente de producción de tiroglobulina sérica, el diagnóstico de sospecha de carcinoma diferenciado de tiroides es altamente sugerente cuando los niveles de tiroglobulina se encuentran elevados, a pesar de que se pueden encontrar elevados en la tirotoxicosis, tiroiditis y deficiencia de yodo (3), pero estos niveles no se modifican en el carcinoma medular ni en el anaplásico siendo también independientes de los de TSH.

La punción con aguja fina es negativa en un $40 \%$ de los casos (4). En nuestro caso no se pudo realizar el diagnóstico anatomopatológico. Decir que la mayor parte de los tumores tiroideos malignos corresponde a carcinoma papilar, que también son los que tienen mejor pronóstico (5), con una mortalidad muy baja (6). 
El cáncer diferenciado del tiroides se disemina por vía linfática, pudiendo aparecer metástasis pulmonares y óseas. En estudios realizados aparecieron metástasis a distancia en el $8 \%$ de los papilares, en el $11 \%$ de los foliculares el $50 \%$ de los medulares (7). En algunos casos la primera manifestación puede ser el hallazgo de una metástasis de forma casual (8). Debido al lento crecimiento de estas metástasis su sintomatología puede ser muy escasa, a veces las metástasis pulmonares pueden producir la muerte debido al compromiso aéreo que producen (9).

Los pacientes con metástasis pulmonares que no reciben tratamiento con radioiodo tienen una vida media a los 5 años del $30 \%$, mientras que los pacientes que siguen tratamiento con iodo es del $60 \%(10)$.

\section{F. J. Nicolás Sánchez, M. E. Nicolás Sánchez², M. T. Merino Laborda, M. L. Espinel Garuz ${ }^{1}$}

Servicio de Medicina Interna, ${ }^{\prime}$ Radiología $y{ }^{2}$ Medicina de Familia. Hospital Val d'Arán. Vielha (Lleida)

1. Fiegge J. Epidemiology of thiroid cancer. In: Wartoksky L, ed. Thyroid Cancer: a comprehensive guide to clinica management. Totowa: Humana Press, 1999; 77-83.

2. Hundahl SA, Fleming ID, Fremgen AM, Menk HR. Anational Cancer Data Base report on 53.856 cases of thyroid carcinoma treated in the US, 1985-1995. Cancer 1998; 83: 2638-2648.

3. Baskin HJ. Thyroglobulin: a clinical review. Endocr Pact 1995; I: $365-$ 367.

4. Hundahl SA, Cady B, Cunningham MP, et al. Initial results from a prospective cohort study of 5.583 cases of thyroid carcinoma treated in the Unites States during 1996. U.S. and German Thyroid Cancer Study Group. Evaluation Study. Cancer 2000; 89: 202-217.

5. Guilland FD, Hunt WC, Morris DM, Key CR. Prognostic factors for thyroid carcinoma. A population-based study of 15.698 cases from the Surveillance, Epidemiology and End Results (SEER) program 19731991. Cancer 1997; 79: 564-73.

6. Hay ID, Grant CS, van Hereden JA, Goellner JR, Ebersold JR, Bergstralh EJ. Papillary thyroid microcarcinoma: a study of 535 cases observed in a 50-yeard-period. Surgery 1992; 112: 1139-47.

7. Alcázar V, Mondéjar BM, del Val T L, de Icaya P M, del Olmo D, Jaunsolo MA, Vázquez C. Tasa de incidencias y características del cáncer de tiroides en un área de la zona sur de Madrid. Endocrinol 2000; 47: 182184.

8. Higashiyama M, Kodama K, Yokouchi H, et al. Mediastinal lymph node involvement as the initial manifestation of occult thyroid cancer in the surgical treatment of lung cancer: report of a case. Surg Today 1999; 29: 670-4.

9. Kitamura Y, Shimizu K, Nagahama M, et al. Immediate causes of death in thyroid carcinoma: clinicopathological análisis of 161 fatal cases. J Clin Endocrinol Metab 1999; 84: 4043-4049

10. Sisson JC, Giordano TJ, Jamadar DA, et al. 131-I treatment of micronodular pulmonary metastases from papillary thyroid carcinoma. Cancer 1996; 78: 2184-92.

\section{Dolor abdominal e inestabilidad hemodinámica en paciente con pluripatología}

\section{Sr. Director:}

La causa más frecuente del hematoma retroperitoneal es la traumática, tras procedimientos invasivos o traumatismo lumbar. Entre las causas no traumáticas la más frecuente es la rotura de aneurisma aórtico. Existen una serie de causas espontáneas entre las que se encuentran las alteraciones de la coagulación, la rotura renal espontánea, los tumores renales y suprarrenales y las enfermedades vasculares (vasculitis y entre ellas la poliarteritis nodosa) $(1,2)$.

Mujer de 83 años con antecedentes de hipertensión arterial, fibrilación auricular paroxística en tratamiento antiagregante, insuficiencia renal crónica y angiodisplasia de colon. Acude a urgencias por episodio de mareo e inestabilidad con incapacidad para emitir lenguaje durante 4 horas. Además presenta cuadros de opresión epigástrica y disnea de esfuerzo progresiva. La paciente ingresa con el diagnóstico de accidente isquémico transitorio y cardiopatía isquémica y se inicia tratamiento con niratos, enoxaparina subcutánea a dosis de $80 \mathrm{mg}$ cada 12 horas, amlodipino y ácido acetil salicílico, quedando asintomática. Entre las pruebas complementarias al ingreso destacan: hemograma y coagulación normales, bioquímica: creatinina $2,3 \mathrm{mg} / \mathrm{dL}$, urea $73 \mathrm{mg} / \mathrm{dL}$, con iones y enzimas cardiacas normales. Electrocardiograma: fibrilación auricular a 100 latidos por minuto. Ecocardiograma: sin alteraciones en la contractilidad. Tomografía Axial Computerizada (TAC) craneal: atrofia cerebral difusa y enfermedad de pequeño vaso. A los 12 días del ingreso la paciente comienza con dolor abdominal, malestar general y vómitos alimentarios. A la exploración, se observan hematomas en los lugares de punción de la heparina, el abdomen es blando, depresible y doloroso a la palpación difusamente, sin signos de irritación peritoneal. Seis horas después presenta inestabilidad hemodinámica sin signos de sangrado digestivo ni sepsis. La paciente desarrolla acidosis metabólica, empeoramiento de la función renal (creatinina $4.5 \mathrm{mg} / \mathrm{dL}$ ), leucocitosis y anemización severa (Hematocrito 21,2\% y $\mathrm{Hb} 6,5$ $\mathrm{mg} / \mathrm{dL}$ ) con APTT 45,6, INR 1.8, actividad de protrombina $43 \%$ y con resto de factores de coagulación normales. Se realiza una TAC abdominal en el que se observa gran hematoma ( 15 × 13 × 8 $\mathrm{cm})$ que se extiende a lo largo de todo el retroperitoneo izquierdo sin afectar espacio perirrenal (Fig. 1). Se practica aortograma con fin diagnóstico y terapéutico y se objetiva sangrado activo procedente de arteria lumbar izquierda L3, realizándose embolización selectiva y colocación de dos espirales metálicos en la arteria (Fig. 2). Se instaura tratamiento de soporte vasoactivo (dopamina), transfusión de hematíes concentrados y plasma fresco y vitamina $\mathrm{K}$ intravenosa. A pesar de lo cual la paciente presenta de nuevo shock hipovólemico con nueva anemización y anuria falleciendo a los 16 días del ingreso. Se practicó autopsia ecográfica estableciéndose los diagnósticos anatomoclínicos de: hemorragia retroperitoneal reciente afectando grasa perirrenal izquierda, miocarditis linfocitaria, pericarditis crónica, cirrosis macromicronodular, nefritis intersticial, nefroangioesclerosis, neumonía bilateral en vías de organización y derrame pleural seroso bilateral.

El hematoma retroperitoneal espontáneo es una complicación grave, aunque infrecuente de la anticoagulación y su incidencia se está viendo aumentada por el incremento en la prescripción de anticoagulantes. A pesar de ello, únicamente entre el 53 y $68 \%$ de los pacientes según distintas series presentan evidencia analítica de antocoagulación excesiva $(3,4)$. En el caso de la enoxaparina, la frecuencia de episodios de sangrado es del $5,2 \%$. Los factores que influyen aumentando el riesgo de sangrado son las altas dosis, la edad avanzada, el daño renal y la utilización concomitante de otros fármacos que alteran la hemostasia (5). Existen varias hipótesis para explicar la fisiopatología de los hematomas espontáneos como la existencia de aterosclerosis difusa en pequeños vasos, vasculopatía de la glándula adrenal, microangiopatía inducida por heparina o traumatismos menores (4). La clínica en la mayoría de los pacientes consiste en dolor abdominal o masa palpable. En un tercio de los pacientes existe inestabilidad hemodinámica. También puede aparecer paresia femoral y ocasionalmente equimosis periumbilical o en flancos. El diagnóstico se suele conseguir con estudios de imagen: ecografía, TAC o angiografía (2). El tratamiento consiste en revertir y suspender la anticoagulación, reposición de volumen y medidas de soporte. La cirugía se reserva para los casos de colapso hemodiná- 
mico no controlado, pero su utilidad está limitada por la localización del punto de sangrado $(3,4)$. La embolización tiene poco valor ya que se cree que la entidad está causada por patología microvascular difusa, pero puede considerarse una alternativa por tratarse de una técnica menos agresiva. En algunos casos se puede realizar drenaje percutáneo según la accesibilidad de la zona $(4,6)$. Nuestra paciente además tenía una hepatopatía crónica no conocida que contribuyó a la persistencia del sangrado que motivó el fallecimiento.

\section{R. Torres-Gárate, E. Álvarez-Rodríguez, N. Bilbao Ormazabal, R. Ciguienza, J. Pérez Pérez, C. Millana', J. Fariña', C. Lozano Tonkín}

Servicios de Medicina Interna I y ${ }^{\prime}$ Anatomía Patológica. Hospital Clínico San Carlos. Madrid

1. Maxit MJ, Paz R. Causas inusuales de hematoma perirrenal y retroperitoneal. Rev HPC 2000; 3: 18-22.

2. Machuca J, Julve E, Galacho A, Pérez D, Quinonero A, Alonso JM, Marin JA. Hematoma retroperitoneal espontáneo: nuestra experiencia. Actas Urol Esp 1999; 23: 43-50.

3. González C, Penado S, Llata L, Valero C, Riancho JA. The clinical spectrum of Retroperitoneal Hematoma in anticoagulated patients. Medicine 2003; 82: 257-62.

4. Sharafuddin MJ, Andersen KJ, Sun S, Lang E, Stecker MS, Wibbenmeyer LA. Spontaneous extraperitoneal hemorrhage with hemodynamic collapse in patients undergoing anticoagulation: Management with selective arterial embolization. J Vasc Interventi Radiol 2001; 12: 1231-4.

5. Melde SL. Enoxaparin-induced retroperitoneal hematoma. Ann Pharmacother 2003; 37: 822-4.

6. Zapzalka DM, Thompson HA, Borowsky SS, Coleman.Steenson CC, Mahowald ML, O'Connell KJ. Polyarteritis nodosa presenting as spontaneous bilateral perinephric hemorrhage: Management with selective arterial embolization. J Urology 2000; 164: 1294-5.

\section{Derrame pleural neoplásico infectado por Salmonella enteritidis}

\section{Sr. Director:}

Las infecciones por salmonella fuera del tracto gastrointestinal son poco comunes aunque en los últimos años han ido cobrando importancia en el paciente inmunocomprometido por su alta incidencia en pacientes con SIDA (1). Los empiemas por Salmonella son extremadamente raros (2-7). Presentamos un caso de derrame pleural de origen neoplásico infectado por Salmonella enteritidis.

Mujer de 76 años que ingresa por síndrome general y dolor lumbar. Entre sus antecedentes destacaba la presencia de diabetes del adulto controlada con antidiabéticos orales, fibrilación auricular en tratamiento con digital e hipertensión arterial en tratamiento con captopril. Ingresa por síndrome general de 1 mes de evolución y dolor lumbar en los últimos días irradiado hacia el abdomen. La exploración era anodina salvo por la presencia de hipoventilación en la base pulmonar izquierda y abdomen ligeramente doloroso en fosa ilíaca izquierda. Afebril durante todo el ingreso. En la analítica se objetiva la presencia de 13.100 leucocitos sin desviación izquierda con serie roja y plaquetas normales y glucemia basal de $207 \mathrm{mg} / \mathrm{dl}$. En la radiografía de tórax al ingreso se observa un pequeño derrame pleural izquierdo, con bioquímica del líquido pleural: 3.200 hematíes, 11.440 leucocitos (45 linfocitos y 55 segmentados), proteínas de 3,6 g/dL (sue- ro: $5,9 \mathrm{~g} / \mathrm{dL}$ ), colesterol de $76 \mathrm{mg} / \mathrm{dL}$ (suero: $79 \mathrm{mg} / \mathrm{dL}$ ), $\mathrm{LDH}$ $1.229 \mathrm{UI} / \mathrm{L}$ (suero: $1.139 \mathrm{UI} / \mathrm{L}$ ) y glucosa: $78 \mathrm{mg} / \mathrm{dL}$. La citología del líquido pleural, repetida en dos ocasiones, fue positiva para adenocarcinoma. Se realizó tomografía computarizada abdominal donde se aprecian imágenes suprarrenales bilaterales sugestivas de metástasis. La paciente sufre un deterioro neurológico brusco en relación con la presencia de metástasis cerebrales, lo que ocasiona su fallecimiento. En el líquido pleural se cultivó Salmonella enteritidis. La paciente no tenía historia de diarrea y los cultivos de orina, heces y sangre fueron negativos.

Es conocida la relación existente entre inmunosupresión e infecciones extraintestinales por salmonella, habiéndose descrito dicha relación en pacientes con tumores sólidos y hematológicos, lupus eritematoso sistémico (LES), trasplante renal, sida y pacientes tratados de forma crónica con corticoides $(1,2)$. Los empiemas por salmonella son extremadamente raros como lo demuestra el hecho de que en una serie de 6.250 casos de salmonellosis documentados bacteriológicamente, solamente existían 3 casos con pioneumotórax, 2 por $S$. typhimurium y 1 por $S$. typhi (3). Se han publicado algunos casos aislados en pacientes con enfermedades neoplásicas diseminadas y así Carel y cols. (4) describen un empiema por salmonella en una paciente con carcinoma tiroideo diseminado y Gill y cols. (5) describen un caso similar en un paciente con carcinoma pulmonar microcítico diseminado. Se publicaron otros casos no relacionados con enfermedades neoplásicas y atribuidos a abscesos subfrénicos $(6,7)$, infecciones tifoideas septicémicas y en algunos casos en pacientes con enfermedades de base sistémicas y/o pulmonares (tuberculosis antigua, enfermedad pulmonar obstructiva crónica y silicosis) $(1,8)$. Llama la atención en nuestro caso la escasa expresividad clínica, no habiéndose asociado el cuadro clínico con salmonellosis sistémi$\mathrm{ca}$, gastroenteritis por salmonella o absceso por salmonella adyacente. En cuanto a la patogenia de las infecciones pleuropulmonares por este germen se han mencionado como posibles mecanismos etiopatogénicos la depresión de la inmunidad, las infecciones en la proximidad, la aspiración de secreciones gástricas infectadas y la vía hematógena $(1,7,9)$. Dada la ausencia de clínica de gastroenteritis y la ausencia de coprocultivos positivos, también se ha pensado en la posibilidad de que existan bacilos en estado latente en el sistema retículo endotelial durante mucho tiempo tras una infección primaria que podrían resurgir cuando se lesiona la función de las células de este sistema por una inmunosupresión importante como puede atribuirse en nuestro caso a la extensión de la enfermedad neoplásica (1). En relación con el tratamiento, éste sería similar al de empiemas por cualquier otro tipo de germen, aplicándose los criterios de drenaje de forma similar. La mortalidad, fue muy elevada en las series consultadas, siendo superior en los pacientes mayores y en aquellos con neoplasias diseminadas $(60-70 \%)(2,4)$ como ocurría en nuestro caso.

\section{García Clemente, F. Hidalgo García ${ }^{1}$, T. González Budiño}

Sección de Neumología. 'Servicio de Microbiología. Hospital Álvarez-Buylla. Mieres. Asturias

1. Aguado JM, Obeso G, Cabanillas JJ, Fernández-Guerrero M, Ales J. Pleuropulmonary infections due to nontyphoid strains of Salmonella. Arch Intern Med 1990; 150:54-56.

2. Wolfe MS, Louria DB, Armstrong D, Blevins A. Salmonellosis in patients with neoplastic disease. A review of 100 episodes at Memorial Cancer Center over a 13 year period. Arch Intern Med 1971; 128: 546554.

3. Lalitha MK, John R. Unusual manifestations of salmonellosis - a surgical problem. Q J Med 1994; 87: 301-309.

4. Carel RS, Schey G, Ma'ayan M, Bruderman I. Salmonella empyema as a complication in malignant pleural effusion. Respiration 1977; 34: $232-5$. 
5. Gill GV, Holden A. A malignant pleural effusion infected with Salmonella enteritidis. Thorax 1996; 51: 104-105.

6. Charles TJ, Hariraj R. Salmonella empyema following splenic biopsy. Respir Med 1998; 92: 968-9.

7. Remarcha Esteras MA, Parra Parra I, Santos Calderón JA. Empiema pleural por Salmonella enteritidis. Arch Bronconeumol 2002; 38: 341.

8. Ruiz M, Rodríguez JC, Elía M, Royo G. Infecciones extraintestinales producidas por serotipos no ifoideos de Salmonella. Experiencia de nueve años. Enferm Infecc Microbiol Clin 2000; 18: 219-222.

9. Berdonces P, Echevarría MJ, Medoza F, López de Goicoechea MJ, Ayarza R. Empyema due to salmonella enterica serotype enteritidis. Enferm Infecc Microbiol Clin 2001; 19: 238.

\section{Mesenteritis retráctil asociada a linfoma difuso en una paciente de 74 años}

\section{Sr. Director:}

La mesenteritis retráctil (MR) o paniculitis retráctil es un proceso poco frecuente de etiología no clara caracterizada por inflamación, fibrosis y necrosis del mesenterio que suele debutar como masa abdominal con imagen radiológica muy típica. Su asociación con linfomas es inusual. Presentamos el caso de una paciente mujer de 74 años con esta entidad en el contexto de un linfoma tipo B.

Mujer de 74 años sin antecedentes personales de interés que consultó por dolor epigástrico con irradiación a hipocondrio derecho junto con náuseas y vómitos alimentarios de 15 días de evolución. Refería así mismo febrícula y diarrea con 6-8 deposiciones líquidas, heces acólicas sin productos patológicos y astenia, anorexia y pérdida de aproximadamente $8 \mathrm{~kg}$ de peso en los últimos 6 meses. En la exploración presentaba un estado general conservado con coloración normal de piel y mucosas y temperatura de 37,9 ${ }^{\circ} \mathrm{C}$. La clavícula izquierda mostraba un engrosamiento en tercio medio, doloroso a la palpación y que la paciente refería de dos meses de evolución. Existía una disminución del murmullo vesicular bilateral más marcada en base izquierda. Llamaba la atención la presencia de una masa en mesogastrio de aproximadamente $8 \mathrm{~cm}$ de diámetro de consistencia dura con dolor a la palpación profunda en epigastrio e hipocondrio derecho. El resto de la exploración fue normal. Los análisis destacaban un hemograma y una coagulación normales; creatinina de 2,20 y urea de $81 \mathrm{mg} / \mathrm{dl}$, con iones en sangre normales; hiperbilirrubinemia de $1,87 \mathrm{mg} / \mathrm{dl}$; amilasa y GPT normales. Una radiografía de tórax mostró un derrame pleural que ocupaba el tercio inferior del parénquima pulmonar izquierdo. Se realizó una ecografía abdominal que mostró dos quistes simples hepáticos de pequeño tamaño; vesícula biliar con cálculos en su interior; colédoco muy discretamente aumentado de tamaño; riñones de morfología y tamaño normal; derrame pleural bilateral mayor en lado izquierdo; $y$, en zona centroabdominal, adyacente a vena mesentérica superior, una imagen hipoecogénica de unos $2,5 \times 2 \mathrm{~cm}$ de diámetro sugerente de conglomerado adenopático, sin poder descartar masa a nivel del proceso uncinado pancreático. Se practicó toracocentesis diagnóstica obteniendo un líquido pleural con $2430 \mathrm{cel} / \mathrm{mm}^{3}$ de predominio mononuclear con proteínas de $27 \mathrm{~g} / \mathrm{L}, \mathrm{LDH} 1029 \mathrm{mU} / \mathrm{mL}$ y ADA de 53 U/L. Los cultivos y baciloscopias de este líquido fueron negativos y la citología mostró células mesoteliales reactivas e intenso exudado inflamatorio mixto de predominio linfocitario muy reactivo. Se realizó TAC toracoabdominal con los siguientes hallazgos: engrosamiento de partes blandas en la zona anterior de la clavícula izquierda con adenopatías supraclaviculares; adenopatías mediastínicas y derrame pleural bilateral con atelectasia pasiva del lóbulo inferior y con captación de pleura visceral en probable relación con derrame neoplásico; áreas hiperdensas y de captación irregular en riñones y ambas suprarrenales y reagrupamiento de estructuras mesentéricas de aspecto masivo que engloba estructuras vasculares y muestran pequeñas adenopatías constituyendo la imagen radiológica típica de una "mesenteritis" o "paniculitis" retráctil (Fig. 1). Se realizó biopsia de la masa de partes blandas adyacente a la clavícula que fue diagnóstica para Linfoma difuso de células grandes inmunofenotipo B con alto índice de proliferación. Durante su ingreso la paciente presentó deterioro progresivo de su estado general con empeoramiento de la función renal y oligoanuria que precisó de dos sesiones de diálisis así como anemización progresiva. Se planteó transfusión de hemoderivados y quimioterapia que la paciente rechazó por su condición de testigo de Jehová, falleciendo un mes después de su ingreso.

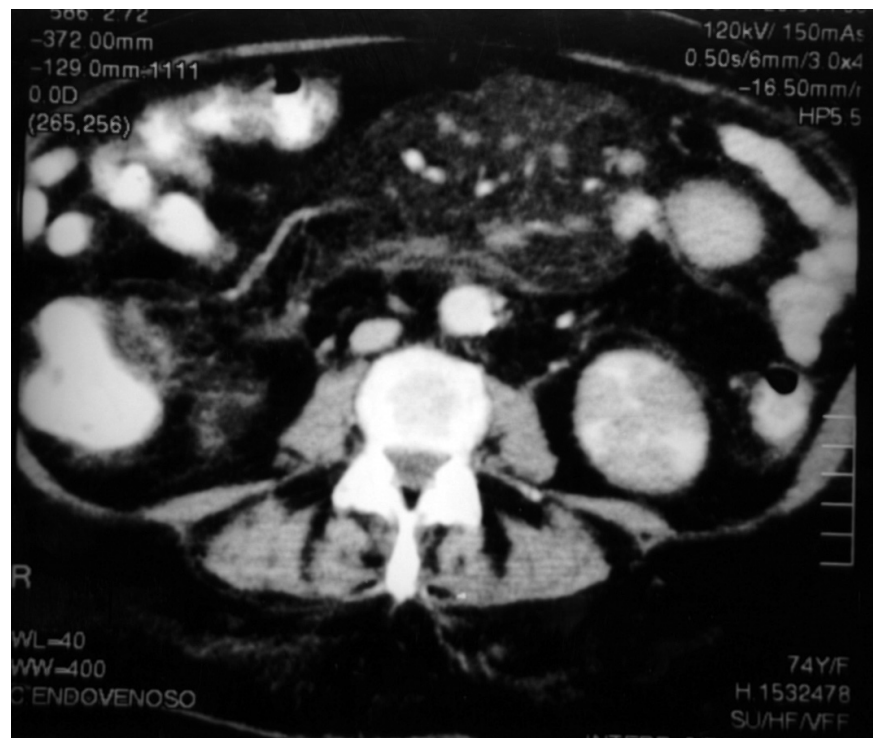

Fig. 1.

La MR es una entidad caracterizada por inflamación, fibrosis y necrosis del mesenterio que fue descrita por primera vez en 1924 (1). Existen pocas referencias en la literatura en relación con este proceso. No hay más de 200 casos descritos (2). Su etiología no está clara, presentándose con más frecuencia en mujeres en la sexta década de la vida (2). Se manifiesta habitualmente en forma de dolor abdominal junto con masa palpable asociado a sintomatología digestiva como diarrea o suboclusión intestinal y síntomas sistémicos tales como febrícula, astenia y pérdida de peso (3). Suele asociarse a entidades benignas como traumatismos abdominales, cirugía previa, enfermedades sistémicas como el lupus eritematoso e infecciones peritoneales como la tuberculosis. Su asociación con neoplasias como el linfoma es muy infrecuente. Hemos realizado una búsqueda en MEDLINE cruzando como palabras clave mesenteritis retractile, lymphoma, paniculitis encontrando una sola referencia en la literatura en este sentido (4). Su diagnóstico definitivo se realiza mediante histología de la lesión que muestra necrosis del tejido graso del mesenterio con fibrosis y componente inflamatorio linfocitario crónico (5). Sin embargo la TAC, ha supuesto un avance en el diagnóstico de este proceso, mostrando habitualmente una imagen de masa localizada de densidad grasa con áreas de menor densidad en su interior, engrosamiento del mesenterio y de la pared intestinal y, ocasionalmente, calcificaciones $(6,7)$. En el presente caso no se realizó laparotomía exploradora ante la existencia de una lesión accesible a punción en clavícula que facilitó el diagnóstico de Linfoma asociado, la ausencia de oclusión intestinal, y la negativa del paciente por su condición de testigo de Jehová a recibir hemoderivados que contraindicaba una cirugía como la referida. Generalmente la evolución de la MR es benigna con tratamiento esteroideo, inmunosu- 
presor o progesterona asociados o no a cirugía, aunque existen casos de mala evolución por obstrucción linfática y de vasos sanguíneos con malabsorción secundaria severa (2). El desenlace fatal de nuestro caso vino condicionado por el fracaso renal atribuido a infiltración por células tumorales que obligó a realización de diálisis que hubo de suspenderse ante la anemización de la paciente y la mala tolerancia a las sesiones con episodios de hipotensión y presíncope, junto con su rechazo a transfusión de sangre.

\section{Grilo Bensusan, I. Vallejo Maroto, R. Maisanaba, E. Pamies Andreu}

Servicio de Medicina Interna. Hospitales Universitarios Virgen del Rocío. Sevilla

1. Jura V. Sulla mesenterite retrattile esclerosante. Policlinico (Prat) 1924; 31: 575-81.

2. Poniachik J, Smok G. Retractile mesenteritis. Report of four cases. Rev Med Chile 2000; 128: 1250-54.

3. Emory TS, Monihan JM, Carr NJ, Sobin LH. Sclerosing mesenteritis, mesenteric panniculitis and mesenteric lipodystrophy: a single entity? Am J Surg Pathol 1997; 21 (4): 392-8.

4. Schwery S, Hangartner PJ, Vollrath T, Buhler H. Mesenteritis paniculitis. Schweiz Med Wochenschr 1993; 123 (25): 1307-11.

5. Ikoma A, Tanaka K, Komokata T, Ohi Y, Taira A. Retractile mesenteritis of the large bowel: report of a case and review of the literature. Surg Today 1996; 26 (6): 435-8.

6. Ueda D. Chiba S. Retractile mesenteritis in a 12-year-old girl: CT findings. Pediatr Radiol 1997; 27: 342-44.

7. Hamrick-Turner JE, Chiechi MV, Abbitt PL, Ros PR. Neoplastic and inflammatory processes of the peritoneum, omentum, and mesentery: diagnosis with CT. Radiographics 1992; 12 (6): 1051-68.

\section{Endocarditis sobre cable de marcapasos por Staphylococcus schleiferi}

\section{Sr. Director:}

Hemos leído con interés el artículo "Endocarditis infecciosa sobre cable de marcapasos", publicado recientemente en su revista por Marchena Yglesias y cols. (1). Desearíamos comunicar un nuevo caso de endocarditis sobre cable de marcapasos (ECM). La ECM es una complicación infrecuente, con una incidencia entre $0,13-7 \%$ (2).

Los factores que predisponen este tipo de infección son: la diabetes, enfermedades neoplásicas y el tratamiento con inmunosupresores; siendo los Staphylococcus spp. las bacterias aisladas con más frecuencia (2-5).

Presentamos un caso de ECM en un varón de 78 años diabético, hipertenso, alérgico a penicilina y rifampicina, con tuberculosis pulmonar antigua y neolasia de colon intervenida hace 3 años. El paciente era portador de un marcapasos (MP) endocavitario desde hacía 9 años, y había sufrido recambio del generador y sutura de los cables hacía 6 meses. Desde entonces refería dos ingresos por fiebre, que no se pudieron filiar. En la actualidad vuelve a ingresar con fiebre de $38,5^{\circ} \mathrm{C}$, tiritona y eritema-tumefacción cutánea en la zona de la incisión. Analíticamente destacaba 15.200 leucocitos ( $87 \%$ neutrófilos) y proteína C reactiva de $53 \mathrm{mg} / \mathrm{L}$.

En los hemocultivos creció Staphylococcus sensible a la mayoría de los antimicrobianos (b-lactámicos, aminoglucósidos, macrólidos y quinolonas) en 4 de 6 botellas. Se envió al Centro Nacional de Microbiología de Majadahonda, donde se confirmó la identificación y el mismo perfil electroforético por campo pul- sado de todas las cepas remitidas.

En la ecografía transesofágica (ET) se observó una vegetación endocárdica de $12 \times 10 \mathrm{~mm}$ a nivel auricular. Con el diagnóstico de ECM, se instauró tratamiento con Vancomicina $(500 \mathrm{mg} / 12$ horas iv) y gentamicina ( $120 \mathrm{mg} / 12$ horas iv) durante 45 días.

La evolución fue buena, a los 17 días de tratamiento, estaba afebril, con hemocultivos negativos y desaparición de la verruga en la ET de control.

El paciente fué intervenido para la extracción de los cables endocárdicos e implante del MP en región abdominal. En los cables retirados creció Staphylococcus schleiferi con el mismo antibiotipo al aislado en sangre.

Los Staphylococcus spp son los causantes de la mayoría de las ECM $(2-5,7,8)$. En la serie de Meune y cols. de 294 ECM, S. epidermidis y S. aureus causan el $71 \%$ de estas infecciones y sólo el $5 \%$ son debidas a otros Staphylococcus spp.

Staphylococcus schleiferi es una nueva especie de estafilococo descrito por Freney en 1988 (6), forma parte de la flora cutánea axilar, y ha sido en los últimos años cuando se le empieza a atribuir importancia como patógeno responsable de infección de heridas, bacteriemia, endocarditis y meningitis (7-8).

A diferencia de Marchena y cols. donde S. epidermidis es considerado un patógeno causante de ECM (1); en nuestro caso, el hallazgo de $S$. schleiferi en 4 de 6 hemocultivos planteó dudas al clínico sobre su verdadero valor (¿contaminante?) y sólo el aislamiento del mismo estafilococo en el cable del MP confirmó su patogenicidad.

El tratamiento de la ECM debe ser doble: tratamiento antibiótico prolongado junto a la retirada del MP, debido al alto porcentaje de recidiva (3-5).

La baja frecuencia de infección por $S$. schleiferi se debe a la rareza de su aislamiento en humanos y sobre todo a las dificultades en la identificación, ya que pueden confundirse con $S$. aureus, lo que hace que su hallazgo en clínica sea una situación excepcional como la que presentamos.

Agradecimiento: Agradecemos a la Dra. Ana Vindel, del Centro Nacional de Microbiología de Majadahonda, su ayuda en la tipificación de las cepas remitidas.

\section{A. Remacha Esteras, A. Esteban Martín, J. A. Herrera Rubio $^{1}$}

Servicios de Microbiología y ${ }^{1}$ Medicina Interna. Hospital Monte San Isidro. León

1. Marchena PJ, Vañó D, Estévez E, Ruiz MD. Endocarditis infecciosa sobre cable de marcapasos. An Med Interna (Madrid) 2004; 21: 97-98.

2. Klug D, Lacroix D, Savoye C. Systemic infection related endocarditis on pacemaker leads. Clinical presentation and management. Circulation 1997; 95: 2098-107.

3. Meune C, Arnal C, Hermand C, Cocheton JJ. Les endocardites infectieuses sur sondes de pacemaker. Ann Med Interne 2000; 151: 456-464.

4. Arber N, Pras E, Copperman Y, Schapiro J, Meiner V, Lossos I et al Pacemaker endocarditis. Report of 44 cases and review of the literature. Medicine 1994; 73: 299-305.

5. Baños R, Gómez J, Sánchez B, de la Morena G, Simarro E, García del Real F. Endocarditis por cable de marcapaso: análisis de 11 casos. Enferm Infecc Microbiol Clin 2000; 18: 267-270.

6. Freney J, Brun Y, Bes M, Meugnier H, Grimont F, Grimont PA et al. Staphylococcus lugdunensis sp. nov. and Staphylococcus schleiferi sp. nov; two species from human clinical specimens. Int J Syst Bacteriol 1988; 38:168-172

7. Leung M, Nuttall N, Mazur M, Taddei T, Mc Comish M, Pearman J. Case of Staphylococcus schleiferi endocarditis and a simple schme to identify clumping factor-positive staphylococci. J Clin Microbiol 1999; 37: 3353-3356.

8. Hernández JL, Calvo J, Sota R, Agüero J, Garcia-Palomo JD, Farinas MC. Clinical and microbiological characteristic of 28 patients with Staphylococcus schleiferi infection. Eur J Clin Microbiol Infect Dis 2001; 20: 153-8. 